\title{
Optical Technique and Image Processing Application in Microgravity Fluid Physics Based on Ground
}

\author{
Q. Kang $^{*} \quad$ L.Duan $\quad$ L.Hu $\quad$ J. F. Zhang $\quad$ W. R. Hu \\ National Microgravity Laboratory/CAS; \\ Institute of Mechanics, Chinese Academy of Sciences, \\ Beijing 100080, China
}

\begin{abstract}
In this paper, several techniques for fluid field measurement, such as Optical Interferometry and Particle Image Velocimetry (PIV) etc. are introduced. And their applications on the fluid physics experiment of simulation microgravity on the ground in the national microgravity laboratory are presented. To reduce the gravitational effect on the ground, the character sizes researched objects are generally very little. The measuring techniques of fluid mechanics have to be combined with micrology to carry out the measurement of little size.
\end{abstract}

Key words: Microgravity Fluid Physics; Experiment of Fluid Mechanics; Optical Interferometry; Particle Image Velocimetry; IR Temperature Measurement; Thermal Liquid Crystal

\section{Introduction}

With the development of the space science and technology, microgravity science has been paid attention in the world. There are a lot of special rules of mass transfer in microgravity environment. Many secondary effects covered by the gravity on the ground become important process and factors. The microgravity fluid physics relates with space material growth and biology sample preparation, and it is also the basement of the microgravity science application. The phenomena introduced by the gravity, for example buoyancy convection and sedimentation etc., will disappear in microgravity environment. New phenomena inspire scientists interesting in the microgravity science. Space station, airship, agravic airplane and drop tower etc. offer scientists opportunity to study microgravity science. Many scientists in the entire world have already worked on this subject. In recent years, the space experiments of fluid physics have also been done in our country, for example, experimental study on the thermal capillary convection of the two layers fluid in SJ-5 satellite and the experiment of the two-phase flow in the space station. Experimental study on microgravity fluid physics must be done on the ground because of the expensive fees of space experiment and a few chance of space experiment. Many optical methods have been used in the National Microgravity Laboratory of CAS, including interferometer used to study the crystal growth and the surface wave [1-3], and PIV used to study the Marangoni convection and the buoyancy convection [4-7].

\footnotetext{
${ }^{*}$ Corresponding author: Q. Kang,

E-mail: kq@imech.ac.cn /Tel: +86-10-62626158 /Fax:+86-10-62615524
} 


\section{Optical interferometer and PIV technique}

The optical techniques that are non-destructive and for whole flow field have been widely used in our laboratory. A Mach-Zehnder interferometer with phase shifter and image processing system used to measure the concentration, density and temperature has been developed as shown in Fig. 1. And a Michelson interferometer has been used to measure the surface deformation of fluid as shown in Fig. 2.

The Particle Image Velocimetry (PIV) is a powerful tool for measuring the velocity field in the experiment of fluid mechanics. It can record the flow structure in a cross-section simultaneously. PIV technique needs to illuminate a cores-section by using a pulse light sheet, to record the sequence of the particles images by using a CCD camera (or a film), and to use the image matching method of a sub-area template between two frames to obtain the displacement (or velocity) at the each sub-area. The arrangement of our PIV system is shown in Fig. 3.

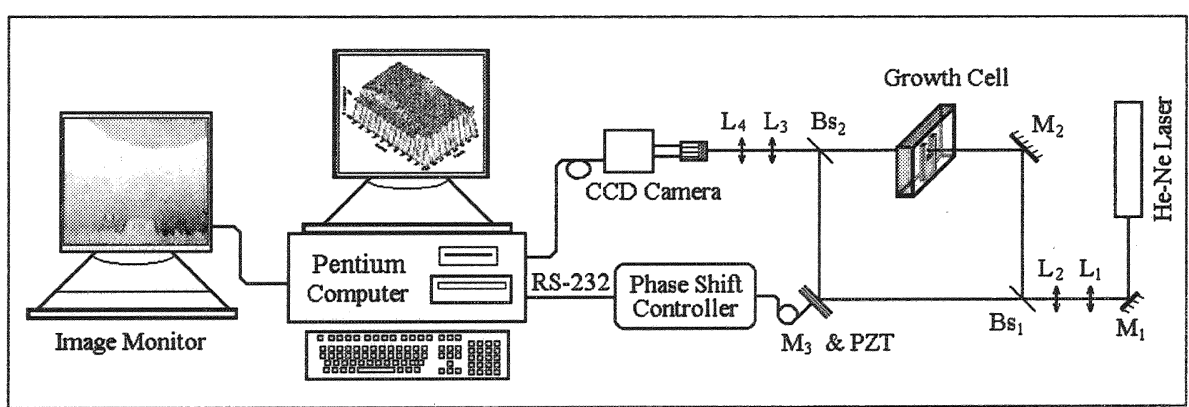

Fig.1 Mach-Zehnder interferometer with phase shifter and image system

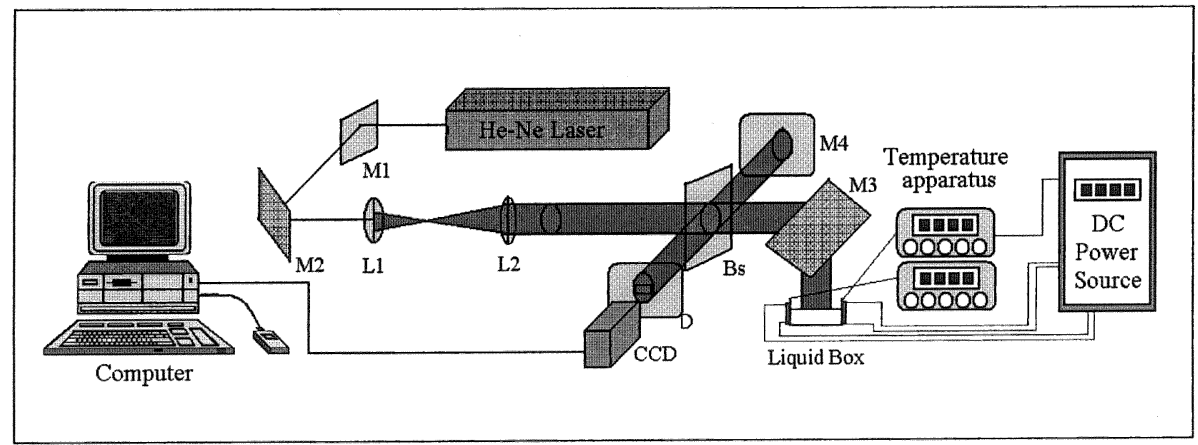

Fig.2 Deformed Michelson interferometer 




Fig.3 Digital particle image velocimetry

\section{Phenomena of the fluid physics in crystal growth}

The crystal growth is a process of heat and mass transfer, which dominates the morphological instability of the growing interface, and it is an important factor for the quality of crystal. The characters of the flow in the crystal growing solution determine the micro-structure of the growing crystal. The property of crystal depends seriously on the solution concentration distribution on the growth surface of a crystal. However, the concentration distributions are affected by the diffusion and convection of the solution. On the ground, the convections caused by buoyancy are generally unavoidable in the crystal growth process from solution. In order to understand and visualize the flow pattern and concentration distribution in the transparent solution, the concentration distributions have been measured by using a micro Mach-Zehnder interferometer and the velocity field was measured by using particle image velocimetry (PIV) in our experiment. The velocity field, concentration field and their relation can be analyzed thereby.

During $\mathrm{NaClO}_{3}$ crystal growth, the solution crystallizes gradually on the surface of the seeding crystal and forms concentration gradient around the crystal. The concentration of the solution around the crystal is smaller than that far away from the crystal, and the direction of the density gradient on the top part of the crystal is directly opposite to that of gravity. Hence, the solution in the diffusion boundary layer of the crystal moves upward in the growth cell, and forms a plume flow in the upper part of the crystal, as shown in Fig. 4. The flow is still a kind of buoyancy convection driven by the exchange of the concentration under the earth's gravity. The flow direction is opposite to the gravity's direction near the crystal. These experimental results show clearly that the mass transfer is carried out mainly by the convection caused by the concentration gradient during the period of the crystal growth. However, the concentration gradient would be reduced because of the convection. The coupling processes of concentration and velocity would influence the rate of the crystal growth.

The convection in the center part above the crystal is mainly vertical to the upper crystal face (010), however, the convection at the two sides of crystal is parallels to two upright crystal faces (100). Therefore, the exchange of solution on crystal faces (100) is more sufficient than that on crystal face (010). The concentration difference in diffusion layer is approximately equal between crystal face (010) and crystal faces (100) as shown in Figure 5, but the gradients of concentration are not equal due to the gravity. That is, the difference of growth rate between the upper crystal face and the side crystal faces is caused by buoyancy convection. 
An argon ion laser was applied to illuminate the flow field of the crystal growth for PIV. Pulse lights were shaped by an electro-optical shutter, which was controlled by a center processor with CCD camera synchronization. The silver-coated hollow glass spheres of $10 \mu \mathrm{m}$ in diameter as tracer
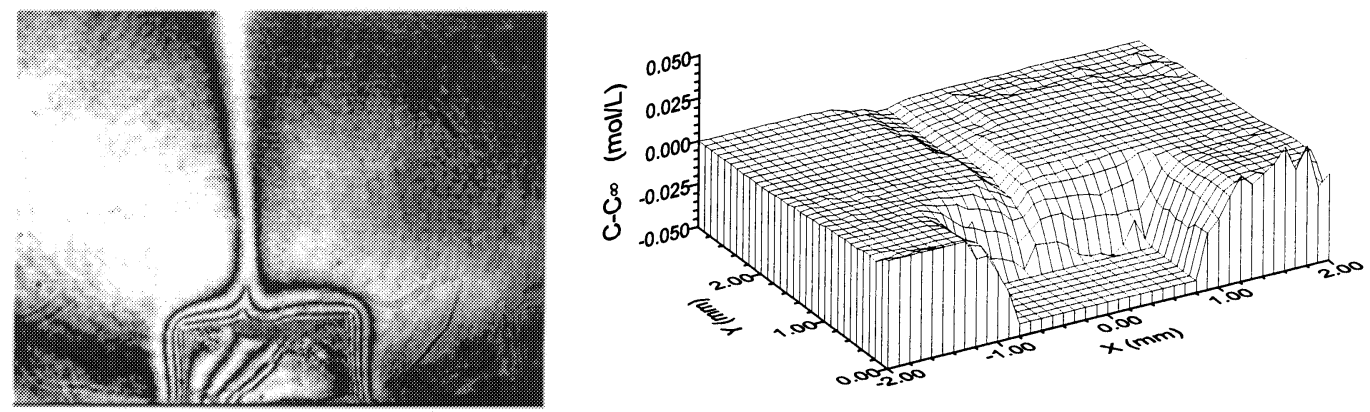

Fig.4 Interference fringe pattern and concentration field in $\mathrm{NaClO}_{3}$ crystal growth

particles are suspended in the $\mathrm{NaClO}_{3}$ solution. The density of the particles is $1.4 \mathrm{~g} / \mathrm{cm}^{3}$, which is close to the density of $\mathrm{NaClO}_{3}$ solution. By using a cycling lens, a $1.0 \mathrm{~mm}$ thin light sheet is shaped to illuminate a vertical cross-section at the center part of the container. A distribution of velocity field is shown in Fig. 6.

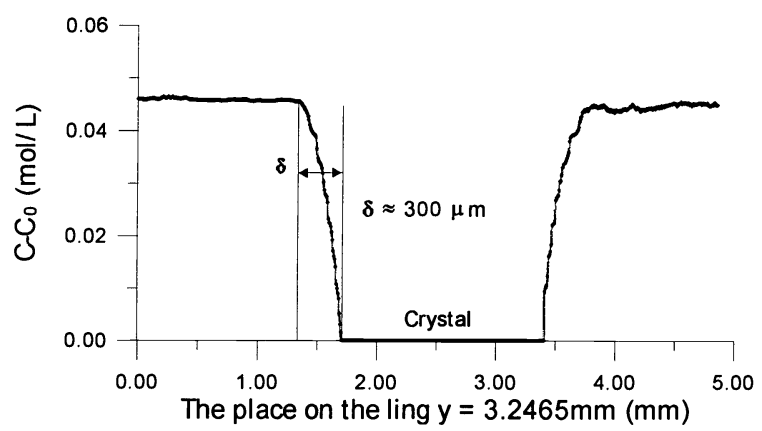

(a) Face (010)



(b) Face (001)

Fig.5 Diffusion layer in $\mathrm{NaClO}_{3}$ crystal growth 

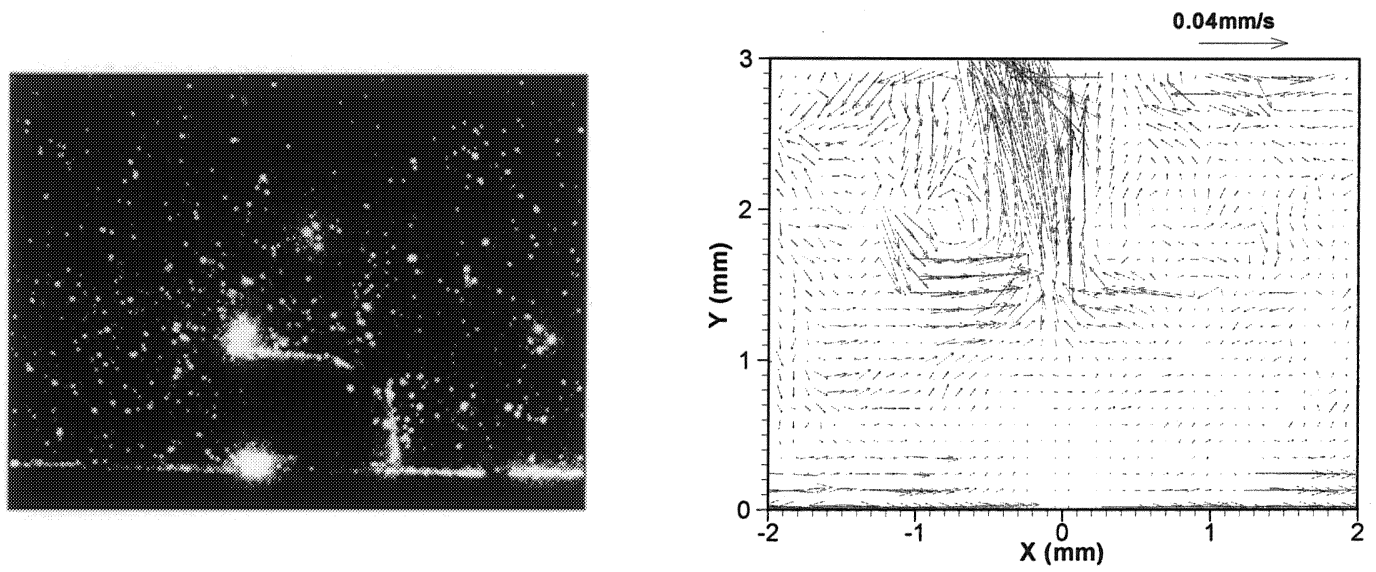

Fig.6 Particle image and Velocity distribution in $\mathrm{NaClO}_{3}$ crystal growth

While the protein crystal grows on the bottom of the growth cell, the interference fringes and the concentration field are shown as in Fig. 7. The curve in Fig. 8 shows the thickness and structure of the concentration boundary layer. The concentration gradient exists only around the growing protein crystal. The gradient is approximately equal at two right angles in present experiment. The results show that there possibly is no buoyancy convection during the process of protein crystal growth. The driving force of protein crystal growth is the diffusion. The result was proved indirectly by another analyses about growth rate [3]. In that paper, the growth rates of the crystal were obtained by analyzing the concentration gradient and the flux of the mass transfer, than the growth rates are compared with those obtained by measuring the size of the crystal directly. The results show that the analyses of the growth rate are very close to the actual growth of the protein crystal. This kind of analyses method could predict the growth rate of the protein crystal by studying the concentration distribution of the solution and shows that the mass transfer during the whole process of the protein crystallization dominates mainly by the diffusion process in comparison with the convection process.
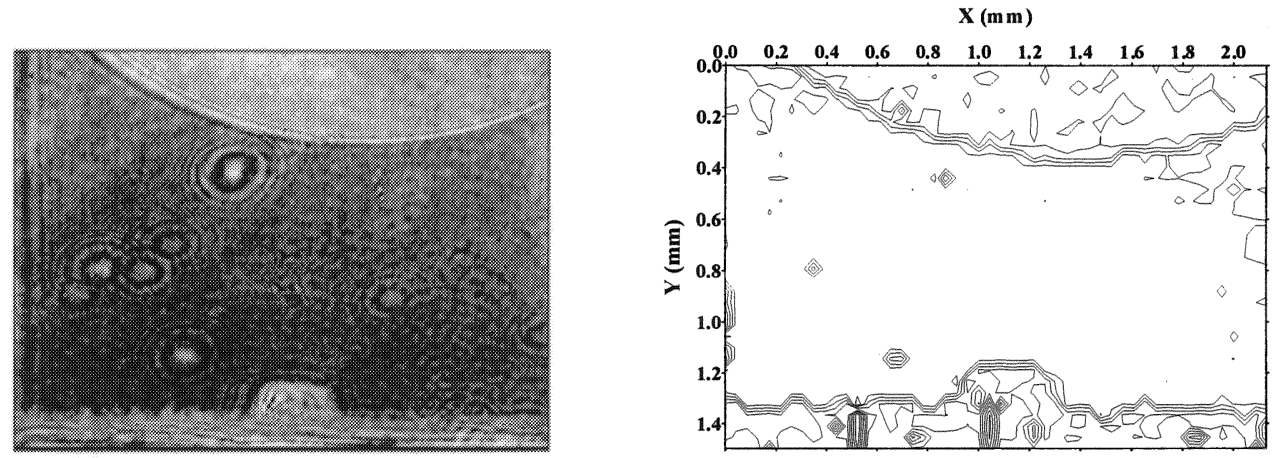

Fig.7 Interference fringe pattern and concentration field in protein crystal growth 


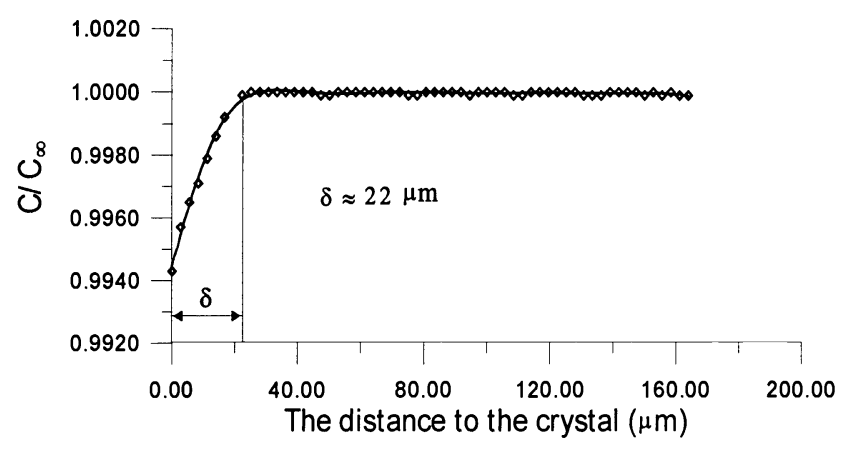

Fig.8 Concentration diffusion layer in protein crystal growth

While the crystal grows on the solution surface, the concentration capillary convection is likely to appear around the growing crystals based on the theoretical analyses. The phenomenon was observed and visualized by using Mach-Zehnder interferometer during the protein crystal growth process as shown in Fig. 9. We can find that the two interference fringes rings appear on the left and right of the crystal near the liquid surface. The convection is a good explain for the experimental image. Without reference to do the experiment in the space or on the ground, the concentration capillary convection always can appear if a crystal grows near the solution surface. Especially in space, it is an important factor to influence crystal growth.
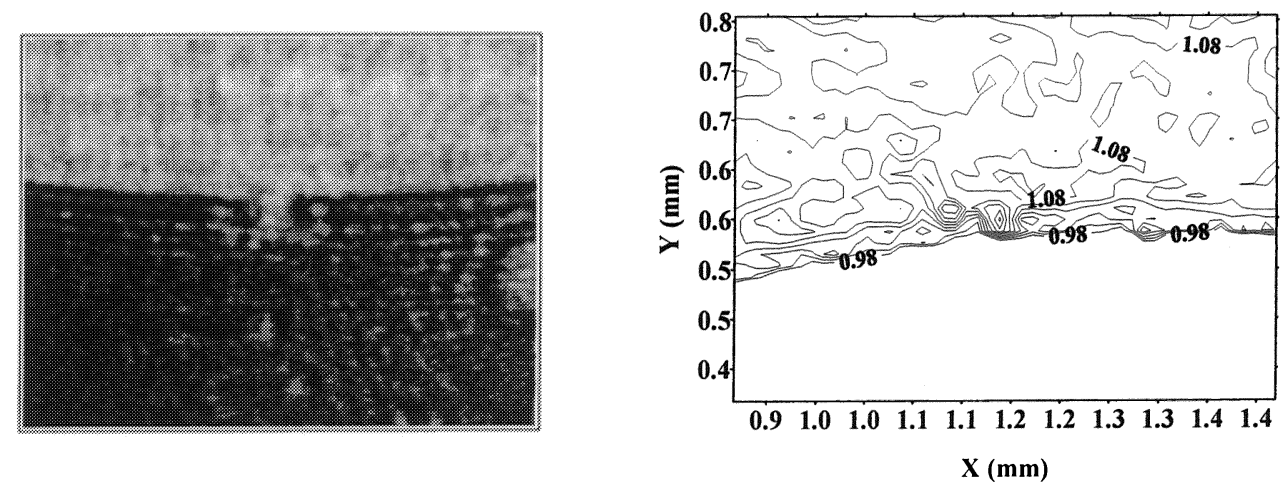

Fig.9 Concentration capillary convection in protein crystal growth

\section{Experimental study on the surface wave}

The thermal capillary convection is driven by the non-uniformity of the surface tension, which comes from the non-uniformity of the surface temperature. It is an important fluid convection phenomenon in many fields, such as crystal growth and film science etc. The surface deformation usually exists if the thermal capillary convection occurs, and the surface wave could appear on the liquid surface when the convection transfers into an oscillatory convection. The surface wave is one of the characteristic processes of flow transition. It is important to study the surface deformation and the surface wave of the thermal capillary convection for understanding the mechanism of the thermal capillary convection. 
In the present work, the technique of the optical interference has been introduced to measure the surface deformation of the fluid layer in a rectangular cavity. The optical diagnostic system consisting of the Michelson interferometer has the better measuring sensitivity in comparison with the conventional experimental method, and the technique of image process has been developed to study the interference fringe images as shown in Fig. 10. Combining this optical interference method with the particle image velocimetry (PIV), not only we can observe the surface deformation and surface wave, but also the velocity field of the thermal capillary convection can be obtained as shown in Fig. 11. The optical interferometer and the PIV technique have been proved to be good means for the experimental study on capillary convection.
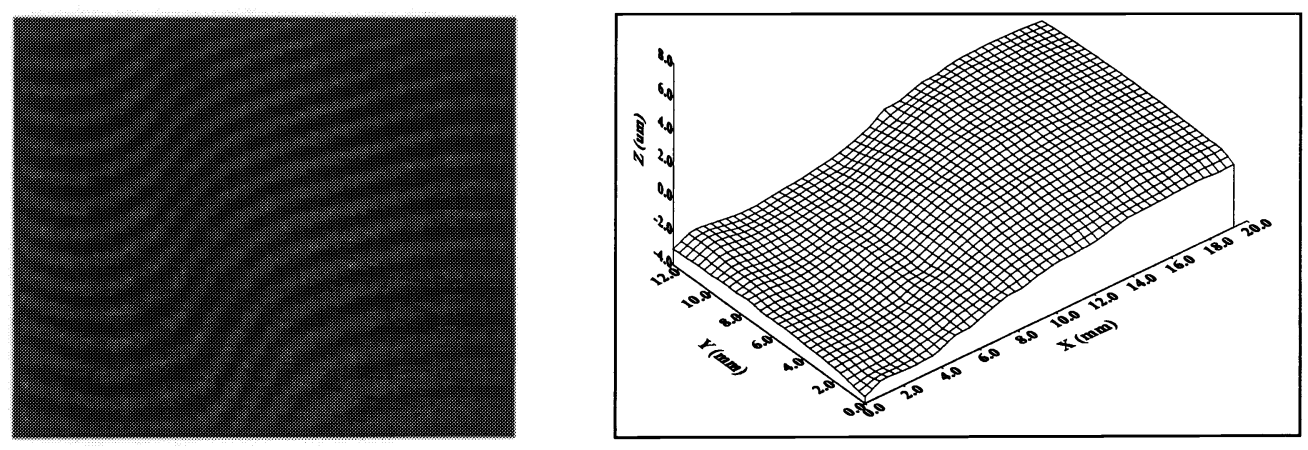

Fig.10 Deformed fringe and corresponding surface shape in the surface wave of thermal capillary



Fig.11 Velocity field of thermal capillary convection

\section{Experimental study on Bénard-Marangoni in single layer liquid}

It is well known that, non-uniformity of the surface tension is the cause of the formation of convective cells in Bénard-Marangoni convection. Besides it refer to the effect of gravitational force on the earth, and the effect of Bénard-convection will be increased with increasing the depth of liquid layer in comparison with the thermocapillary effect.

The experimental investigation of Bénard-Marangoni convection has been performed in a layer of rectangular cavity. The coordinated measurements of velocity distribution in vertical cross-section and temperature pattern on free surface have been obtained respectively using Particle Image Velocimetry 
and IR radiometer in real time. In order to analyze the evolution process of convection cell and the structure of convection vortex, the temperature patterns on the free surface were recorded continuously by using an IR image radiometer of Thermoteknix Systems Model 760. A temperature distribution on the free surface of liquid is shown in Fig. 12. The video frame composite technique was used to record simultaneously the two video signals of two patterns on a cassette with a video recorder. Fig 13 shows a composite image of flow pattern in vertical cross section and the temperature pattern on free surface. The establishment of convective cell is relatively a slow process in the case of very small heating rate. Based on the present experiments, the process from the appearance of the first structure of Bénard cells to the formation of temperature pattern of the complete cells on the whole free surface may be observed clearly. The critical temperature differences were also measured via the detections of both temperature field pattern (IR-imaging) on the free surface and fluid convection (PIV) in the liquid layer. The critical temperature difference or the critical Marangoni numbers were given in ref. 6. The experiments were performed for a fixed depth of air layer and a changeable depth of the liquid layer, and then the influence of the thickness ratio of the air layer to liquid layer on the Marangoni instability was studied [5,6].

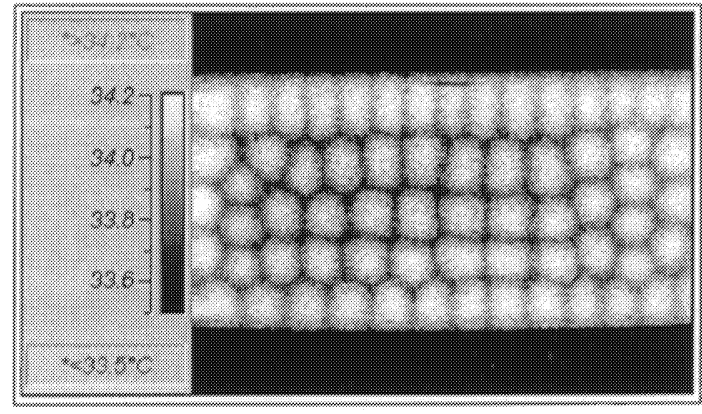

Fig.12 Surface temperature pattern of Bénard cells

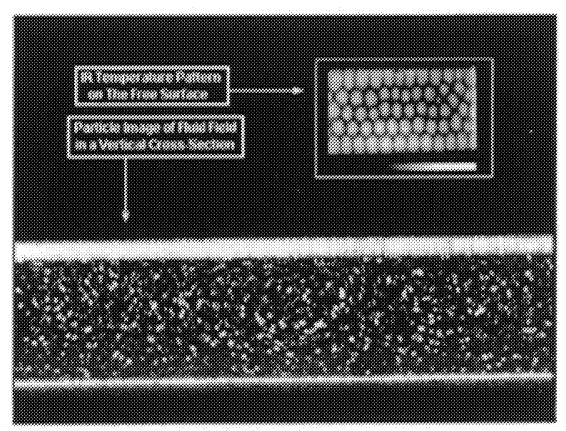

Fig.13 Composite image of flow pattern and temperature pattern

\section{Experimental study on Bénard-Marangoni in double immiscible liquids}

The problem of convection in two superimposed layer of immiscible liquids has received much attention in recent years. There are many theoretical analyses and space experiment research in the field, because there are many kinds of multi-layer liquid phenomena in nature and industry application, and the heat and mass transfer and instability in this kind of system are still not much clear. Specially, not only on the ground but also in space, mantle method of crystal growth has shown a good future. In the two layer of liquid heated from below, the thickness ratio of two liquid layers will become very important to convection instability or convection style. The relationship between critical convection and the thickness ratio of two liquid layers, and the relationship between critical convection and the thickness of two liquid layers are very complex because of simultaneous existence of thermocapillary effect and buoyancy effect. Because of the coupling of buoyancy effect and thermocapillary effect, the convection instability is much more complex than that in the microgravity environment.

The evolution processes of convection have been observed in the differential thickness ratio of two 
liquid layers in present experiments. If the thickness of upper silicon oil layer and the thickness of lower FC-70 layer all are large as shown in Fig. 14, according to the comparison of the Rayleigh number and Marangoni number with their theoretical value of critical convection, onsets of convection of the two liquids all will be induced by the buoyancy effect. However, the onsets of convection are not at the same time in the upper layer liquid and lower layer liquid. Besides, in case of the different thickness of two liquid layers, another typical coupling styles of convection, the coupling of buoyancy convection with Marangoni convection and the coupling of Marangoni convections, were also observed [7]. Fig. 15 shows a color particle image of thermal color liquid crystal (TCL) on the horizontal interface of two liquids. The particle images not only show the flow pattern but also contain the information of temperature distribution, because each hue value of the color images is corresponding to a temperature value. Besides, the velocity field was also obtained from the TCL particle image by means of PIV technique as shown in Fig. 15. That is, combining the TCL with PIV, we can know the flow pattern and temperature pattern on the same area and at the same time.
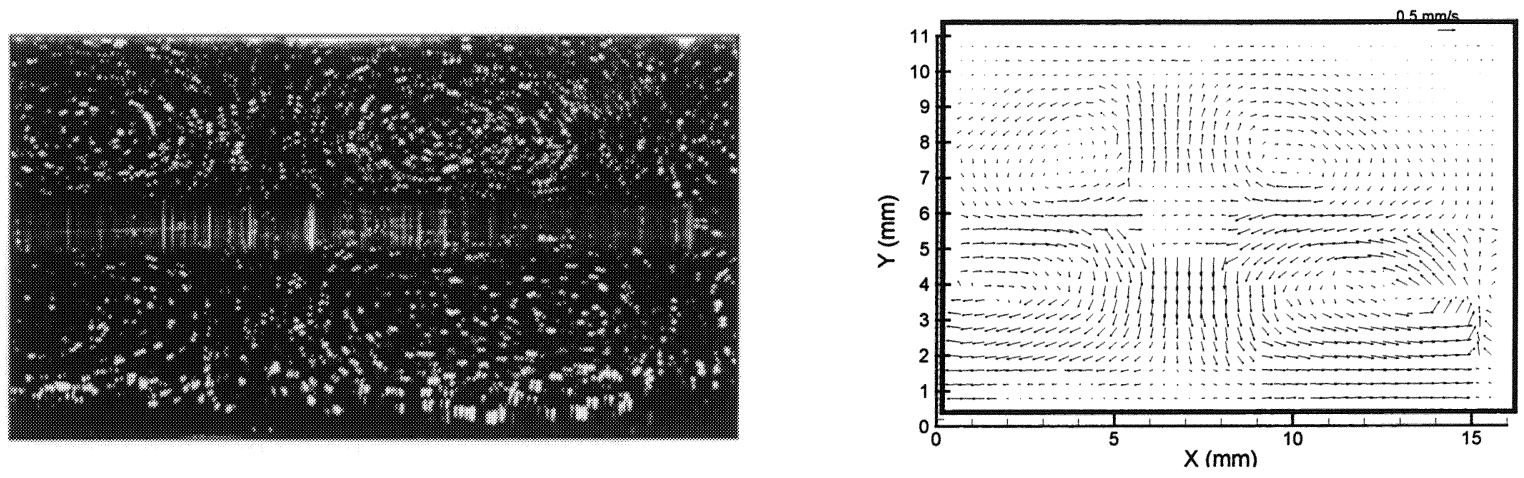

Fig.14 Convection pattern and velocity field of two layers fluid in vertical cross section
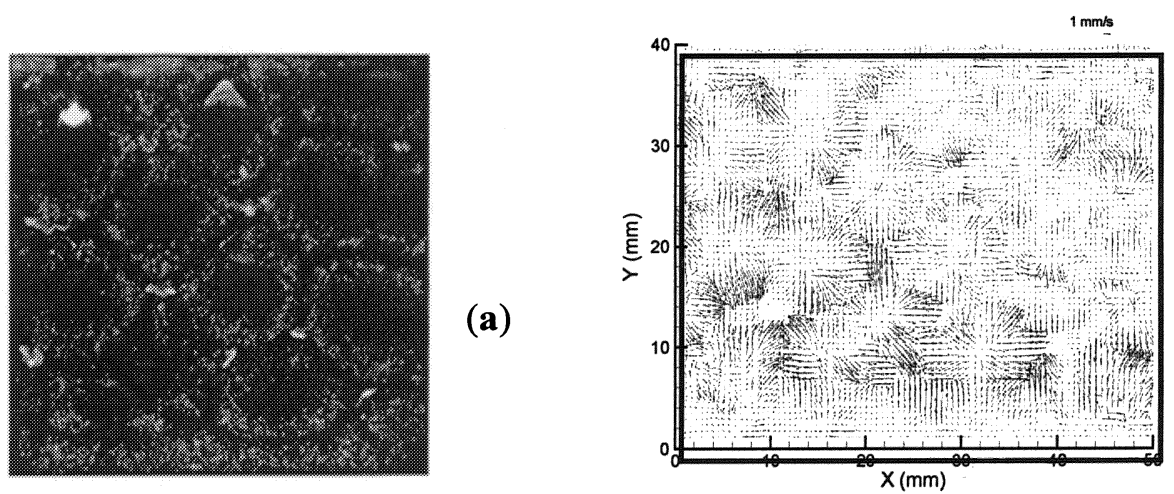

(b)

Fig.15 Convection pattern, temperature distribution and velocity field of two layers fluid in horizontal cross section 


\section{Conclusion}

The advanced experimental system has been developed in National Microgravity Laboratory of CAS in recent years. Many research programs on microgravity science have been studied by using many optical diagnostic techniques. The research works in this paper all were completed in National Microgravity Laboratory of CAS.

\section{References}

1. L. duan, J. Z. Shu, The convection during $\mathrm{NaClO}_{3}$ crystal growth observed by the phase shift interferometer, Journal of Crystal Growth, 223(2001), 181-188

2. L. Duan, Q. Kang, J. Z. Shu, The concentration flow field of the dissolution and crystallization of a mini-crystal studied by the optical diagnostics, Acta Mechanica Sinica, 2002(3), Vol. 34, 463-468

3. L. Duan, Q. Kang, W. R. Hu, G. P. Li, D. C. Wang, The diffusion process and the growth rate in the protein crystal growth, Biophysical Chemistry, 97 (2002), 189-201。

4. Q. Kang, L. Duan, Mass Transfer Process during the $\mathrm{NaClO}_{3}$ Crystal Growth Process, International J. Heat \& Mass Transfer, 44 (2001), 3213-3222

5. Q.S. Liu, Q. Kang, W.R. Hu, The effect of air layer on the Marangoni-Bénard convection of liquid layer in an enclosed cavity, AIAA, IAF-99-J4.06 1996.

6. Q. Kang, W.R. Hu, Influence of the Layer of Air on Bénard-Marangoni Convection, Microgravity science and Technology, XII/2(1999) P61-65.

7. Q. Kang, J.F. Zhang, L. Hu, W.R. Hu, Experimental study on Bénard-Marangoni convection in double immiscible liquid layers, $L A C-02-J .4 .01,2002$. 